\title{
Crosstalk between H1975 tumor cells and platelets to induce the proliferation, migration and tube formation of vascular endothelial cells
}

\author{
BAIKUN LI $^{1 *}$, XINGYU DONG $^{2 *}$, JIMIN ZHU $^{3}$, TING ZHU $^{2}$, XIAOXIAO TAO $^{4}$, DAIYIN PENG $^{4}$ and QINGLIN LI ${ }^{4}$ \\ ${ }^{1}$ School of Traditional Chinese Medicine; ${ }^{2}$ School of Pharmacy; ${ }^{3}$ School of Life Sciences, \\ Anhui University of Chinese Medicine, Hefei, Anhui 230012; ${ }^{4}$ Key Laboratory of Xin'an Medicine \\ (Anhui University of Chinese Medicine), The Ministry of Education, Hefei, Anhui 230038, P.R. China
}

Received February 5, 2021; Accepted June 21, 2021

DOI: $10.3892 / \mathrm{ol} .2021 .12937$

\begin{abstract}
Activated platelets (PLTs) participate in the regulation of tumor angiogenesis, and tumors can activate PLTs. Whether co-culture of lung carcinoma with PLTs improves the function of human umbilical vein endothelial cells (HUVECs) requires further investigation. The present study aimed to investigate the impact of H1975 cell crosstalk with PLTs on the proliferation, migration and tube formation of HUVECs. Following generation of cell-derived supernatants and construction of the co-culture system, Cell Counting Kit-8, flow cytometry, transmission electron microscopy and a meter for epithelial measurement were performed to detect the proliferative ability of HUVECs. Furthermore, the wound healing and Transwell migration assays were performed to detect the migratory ability of HUVECs. A tube formation assay was performed to assess angiogenesis, ELISA was applied to detect the content of vascular endothelial growth factor (VEGF) and western blotting was carried out to measure the expression levels of VEGF receptor 2 (VEGFR2) in HUVECs. Compared with single-cultured HUVECs (control), co-culture with H1975 cells and PLTs (Exp_HP) improved cell proliferation, increased the proportion of cells in the S-phase, destroyed the cell ultrastructure and decreased transepithelial electrical resistance in HUVECs. In addition, a higher relative migration rate, greater number of migrated cells, stronger tube-forming ability and increased expression of VEGF and VEGFR2 were detected in the Exp_HP group compared with the control group. The properties of HUVECs in Exp_H (co-cultured with
\end{abstract}

Correspondence to: Dr Qinglin Li, Key Laboratory of Xin'an Medicine (Anhui University of Chinese Medicine), The Ministry of Education, 103 Meishan Road, Hefei, Anhui 230038, P.R. China

E-mail: liqinglin@ahtcm.edu.cn

\section{*Contributed equally}

Key words: lung carcinoma, platelet, vascular endothelial cells, angiogenesis, co-culture
H1975 cells) were similar to those in Exp_HP, but significantly weaker. Taken together, the results of the present study suggest that tumor cells interacting with PLTs may play an important role in tumor angiogenesis by affecting or mediating changes in the properties of vascular endothelial cells (VECs).

\section{Introduction}

Lung cancer is the most common malignancy overall and accounted for $11.6 \%$ of the total number of cancer cases worldwide in 2018 (1). Furthermore, 80\% lung cancer cases are non-small cell lung cancer (NSCLC), and lung adenocarcinoma is the most common subtype of NSCLC (1). Tumor metastasis is the main cause of clinical treatment failure and mortality (2). Lung adenocarcinoma is a type of lung cancer that easily metastasizes. Most patients with lung cancer who do not smoke suffer from lung adenocarcinoma with epidermal growth factor receptor (EGFR) mutations (3). In China, the proportion of women with lung cancer is increasing, while $\sim 80 \%$ of Chinese women with lung cancer do not smoke (4). Understanding the underlying molecular mechanism of lung adenocarcinoma is useful to improve the therapeutic regimen and effect of treatment, particularly for patients who do not smoke.

Tumor metastasis is a complex pathophysiological process in which vascular microenvironment remodelling plays a key role in the formation of the tumor metastasis microenvironment $(5,6)$. Angiogenesis is an important pathological and physiological basis for the growth and invasion of tumor cells $(7,8)$. Vascular endothelial cells (VECs) play an important role in the process of angiogenesis (9). Studies have reported that the components secreted by tumor cells can stimulate angiogenesis. For example, the outer vesicles derived from osteosarcoma cells can induce angiogenesis (10), and the exosomes secreted by ovarian cancer cell ovacar- 3 can promote the expression and secretion of vascular endothelial growth factor (VEGF) in endothelial cells (ECs), thereby enhancing the proliferative and migratory abilities of ECs (11).

Platelets (PLTs) also play an important role in tumor growth and metastasis (12-14). For example, activated PLTs can encapsulate tumor cells, enhance the ability of tumor cells 
to cope with blood flow shear force and escape the killing of the immune system, mediate the adhesion and extravasation of tumor cells and vascular ECs (15), and participate in the regulation of tumor angiogenesis (16-18). PLTs are activated by tumor cells and exhibit higher expression of pro-angiogenic factors, such as von Willebrand factor, vascular endothelial growth factor (VEGF) and sphingosine-1-phosphate (19). However, antiplatelet therapy often leads to physiological coagulation abnormalities, increasing the risk of bleeding (20). Activated PLTs contain various active biomolecules, which promote the proliferation of tumor cells (20). Thus, it is speculated that the interaction between tumor cells and PLTs can promote the proliferation, migration and tube formation of vascular ECs. However, further investigations are required to confirm this hypothesis.

In the present study, the lung adenocarcinoma cell line, H1975 with EGFR mutation, was isolated from a woman with lung adenocarcinoma who does not smoke. A co-culture in vitro system was used to simulate the interaction between H1975 cells and PLTs and evaluate its impact on the proliferation, migration and tube formation of vascular ECs to lay the foundation for future studies on the mechanism and effect of drug intervention among patients with lung adenocarcinoma who do not smoke.

\section{Materials and methods}

Cell culture. The human lung adenocarcinoma cell line, H1975, and HUVECs were kindly provided by the Cell Bank of Type Culture Collection of the Chinese Academy of Sciences. Cells were maintained in RPMI-1640 medium (Thermo Fisher Scientific, Inc.) supplemented with $1 \%$ penicillin/streptomycin and $10 \%$ fetal bovine serum (FBS; Thermo Fisher Scientific, Inc.), at $37^{\circ} \mathrm{C}$ in a humidified atmosphere with $95 \%$ air and $5 \% \mathrm{CO}_{2}$.

Preparation of PLT. A total of $10 \mathrm{ml}$ of venous whole blood was extracted from healthy adult volunteer, using acid citrate dextrose (15\% v/v; lot, 0803A20; Beijing Leagene Biotechnology Co., Ltd.) as an anticoagulant (21). Following centrifugation at $190 \mathrm{x}$ g for $20 \mathrm{~min}$ at room temperature in a horizontal centrifuge, PLT-rich plasma was obtained by carefully extracting the supernatant, which was centrifuged at $650 \mathrm{x}$ g for $10 \mathrm{~min}$ at room temperature. PLTs were washed in citrate-glucose-sodium buffer (CGS; $14.7 \mathrm{mM}$ trisodium citrate, $33.3 \mathrm{mM}$ glucose, $123 \mathrm{mM} \mathrm{NaCl}, \mathrm{pH}$ 7.0) and centrifuged at $600 \mathrm{x} g$ for $5 \mathrm{~min}$ at room temperature. After washing twice with CGS, PLTs were resuspended using prewarmed RPMI-1640 medium without FBS. Subsequently, a suspension with $3.0 \times 10^{8} \mathrm{PLTs} / \mathrm{ml}$ was made and immediately used for cell experiment (17). All participants provided oral consent after fully explanatory statements of the study.

Generation of cell derived supernatant (SN). A total of two types of supernatants were generated, namely, H1975 cell-derived supernatant (SN_H) and supernatants derived from H1975 cells co-cultured with PLT (SN_HP). Briefly, $\mathrm{H} 1975$ cells were cultured to confluence in a $75 \mathrm{~cm}^{2}$ petri dish, and the culture medium was replaced with RPMI-1640 medium without FBS. Following incubation for $24 \mathrm{~h}$ at $37^{\circ} \mathrm{C}$, the cell supernatant was collected in an aseptic tube and centrifuged at $1,800 \times \mathrm{g}$ for $10 \mathrm{~min}$ at room temperature to eliminate cell debris, and SN_H was subsequently frozen at $-80^{\circ} \mathrm{C}$. Similarly, H1975 cells were cultured to confluence in a $75 \mathrm{~cm}^{2}$ petri dish, and cells were thoroughly washed with PBS following removal of the culture medium. Following incubation with PLT suspension and RPMI-1640 medium without FBS for $24 \mathrm{~h}$ at $37^{\circ} \mathrm{C}$, SN_HP were harvested in aseptic tubes and frozen at $-80^{\circ} \mathrm{C}$ following centrifugation at $1,800 \mathrm{x} \mathrm{g}$ for $10 \mathrm{~min}$ at room temperature. Supernatants were used for the wound healing and tube formation assays, which consisted of three groups: Control stands for single-cultured HUVECs, Exp_H stands for HUVECs co-cultured with SN_H and Exp_HP stands for HUVECs co-cultured with SN_HP.

Construction of the co-culture system (CCS). In total, three groups (control, Exp_H and Exp_HP) were set up, and each group consisted of an upper chamber and a lower chamber (22). For the control group, HUVECs $\left(1 \times 10^{4}\right.$ cells per well for 24-well plates and $1 \times 10^{5}$ cells per well for 6-well plates) were seeded into the upper chambers with RPMI-1640 medium, while RPMI-1640 medium supplemented with 10\% FBS was added to the lower chambers. For the Exp_H group, HUVECs $\left(1 \times 10^{4}\right.$ cells per well for $24-w e l l$ plates and $1 \times 10^{5}$ cells per well for 6-well plates) were seeded into the upper chambers with RPMI-1640 medium, H1975 cells $\left(1 \times 10^{4}\right.$ cells per well for 24-well plates and $1 \times 10^{5}$ cells per well for 6 -well plates) and RPMI-1640 medium supplemented with 10\% FBS were added to the lower chambers. For the Exp_HP group, HUVECs $\left(1 \times 10^{4}\right.$ cells per well for $24-w e l l$ plates and $1 \times 10^{5}$ cells per well for 6-well plates) were seeded into the upper chambers with RPMI-1640 medium, and H1975 cells (1x10 4 cells per well for 24-well plates and $1 \times 10^{5}$ cells per well for 6 -well plates), PLTs ( $2 \times 10^{6}$ cells per well for $24-w e l l$ plates and $2 \times 10^{7}$ cells per well for 6-well plates) and RPMI-1640 medium supplemented with $10 \%$ FBS were added to the lower chambers.

The upper chamber inoculated with HUVECs was transferred to the corresponding lower chamber. Control stands for single-cultured HUVECs, Exp_H stands for HUVECs co-cultured with H1975 cells and Exp_HP stands for HUVECs co-cultured with H1975 cells and PLTs. Based on CCS, cell viability, cell resistance, and Transwell migration measurements were performed using 24-well Transwell culture plates, and morphological observation, cell cycle analysis, VEGF and VEGF receptor 2 (VEGFR2) expression measurements were performed using 6-well Transwell culture plates. The specific process of each detection method is described subsequently.

Detection of PLT activation. PLTs were incubated with SN_H for $2 \mathrm{~h}$ at $37^{\circ} \mathrm{C}$ in a humidified atmosphere with $5 \% \mathrm{CO}_{2}$, centrifuged at $2,000 \mathrm{x}$ g for $5 \mathrm{~min}$ at room temperature, and resuspended in $100 \mathrm{ml}$ PBS. Cells were subsequently incubated in the dark with phycoerythrin (PE) isotype (lot, 12471482; Thermo Fisher Scientific, Inc.) and PE-labelled anti-P-selectin (1:20 dilution; lot, 2265654; Thermo Fisher Scientific, Inc.) at room temperature for $30 \mathrm{~min}$, and subsequently fixed with $1 \%$ paraformaldehyde (PFA) at room temperature for $10 \mathrm{~min}$. Cells were centrifuged at $400 \mathrm{x} \mathrm{g}$ for $5 \mathrm{~min}$ at room temperature and resuspended in $500 \mathrm{ml}$ of PBS. P-selectin was measured using a FACSCalibur flow cytometer (Becton-Dickinson 
and Company) and analyzed using CXP analysis software (version 2.2; Beckman Coulter, Inc.).

Cell Counting Kit-8 (CCK-8) assay. A total of three groups were designed based on CCS. For the control group, $1 \times 10^{4}$ HUVECs were seeded into the upper chambers (Corning, Inc.) with $0.5 \mathrm{ml}$ RPMI-1640 medium, while $1 \mathrm{ml}$ of RPMI-1640 medium supplemented with $10 \%$ FBS was added to the lower chambers. For the Exp_H group, 1x10 $0^{4}$ H1975 cells were seeded into the lower chambers based on the control. For the Exp_HP group, $2 \times 10^{6}$ PLTs were seeded into the lower chambers based on the Exp_H.

The upper chamber inoculated with HUVECs was transferred onto the corresponding lower chamber. Following incubation for 1, 2, 3 and 4 days, CCK-8 reagent (lot, 69112500; Biosharp Life Sciences) was used to detect HUVEC proliferation, and a microplate reader (Molecular Devices, LLC) was used to measure the optical density (OD) at a wavelength of $450 \mathrm{~nm}$ after another $2 \mathrm{~h}$ of incubation with the CCK-8 reagent. The OD values are represented as the mean absorbance for three wells from each group.

Cell cycle analysis. A total of three groups were designed based on CCS. For the control group, $1 \times 10^{5}$ HUVECs were seeded into the upper chambers with $2 \mathrm{ml}$ of RPMI-1640 medium, while $3 \mathrm{ml}$ of RPMI-1640 medium supplemented with $10 \%$ FBS was added to the lower chambers. For the Exp_H group, $1 \times 10^{5} \mathrm{H} 1975$ cells were seeded into the lower chambers at the same time as the control. For the Exp_HP group, $2 \times 10^{7}$ PLTs were seeded into the lower chambers based on the Exp_H.

The upper chamber inoculated with HUVECs was transferred to the corresponding lower chamber. Following incubation for 3 days at $37^{\circ} \mathrm{C}$, cells were washed twice with cold PBS and harvested. Subsequently, cells were treated with ribonuclease A (Rnase A; lot, ST576; Beyotime Institute of Biotechnology) for $30 \mathrm{~min}$ at $37^{\circ} \mathrm{C}$, and then treated with propidium iodide (PI; lot, 7007583; Biosharp Life Sciences) in the dark for $30 \mathrm{~min}$ at $4^{\circ} \mathrm{C}$. The fluorescence intensity of cells was determined using a FACSCalibur flow cytometer (Becton-Dickinson and Company). Cell cycle phase distribution was calculated using ModFit LT software (Verity Software House, Inc.).

Morphological observation. Grouping design and cell culture conditions were the same as those used for cell cycle analysis. Briefly, HUVECs were collected following incubation for 3 days at $37^{\circ} \mathrm{C}$, fixed in $3 \%$ glutaraldehyde (lot, 2191108; Ted Pella, Inc.) for $10 \mathrm{~h}$ at $4^{\circ} \mathrm{C}$, postfixed in $1 \%$ osmium tetroxide (lot, 4008-182802-100118; Ted Pella, Inc.) for $1 \mathrm{~h}$ at $4^{\circ} \mathrm{C}$, dehydrated in graded ethanol at room temperature, and subsequently embedded in Epon. Thin sections were mounted on copper grids, stained with lead citrate (lot: 180705; Ted Pella, Inc.) for $30 \mathrm{~min}$ at room temperature. Transmission electron microscopy (JEM 1400; JEOL, Ltd.) was used to observe the morphological changes in the HUVECs.

Cell resistance measurement. Grouping design and cell culture conditions were the same as those for the CCK- 8 assay. Following incubation for 1, 2, 3 and 4 days, cell resistance was continuously measured using a meter for the epithelium instrument (RE1600, Beijing Jinhongtai Technology Co., Ltd.). The impedance value could be used to reflect the degree of cell resistance, that is, high impedance indicates tight junctions, while low impedance indicates loose junctions (23).

Wound healing assay. Cell suspension containing $1 \times 10^{6}$ HUVECs $(2 \mathrm{ml})$ was added to a 6 -well plate. The monolayer of cells was scratched in the middle with a pipette tip when cells grew to $80-90 \%$ of confluence and washed twice with sterile PBS to remove debris. Cells were subsequently cultured with $2 \mathrm{ml}$ of SN_H, SN_HP and RPMI-1640 medium without FBS to form the Exp_H, Exp_HP and control groups, respectively. The cells were incubated at $37^{\circ} \mathrm{C}$, and the scratches were observed using the CKX41 inverted light microscope (magnification, $\mathrm{x} 40$; Olympus Corporation) at 0 and $12 \mathrm{~h}$. ImageJ software (version 1.52a; National Institutes of Health) was used to measure the gap distance of the wound and compute the relative migration rate.

Transwell migration assay. Grouping design and cell culture conditions were the same as those for the CCK- 8 assay. Briefly, the lower chambers were added with $1 \mathrm{ml}$ of RPMI-1640 medium (10\% FBS) per well for the control group, $1 \mathrm{ml}$ of RPMI-1640 medium (10\% FBS) plus $1 \times 10^{4} \mathrm{H} 1975$ per well for the Exp_H group, $1 \mathrm{ml}$ of RPMI-1640 medium (10\% FBS) plus $1 \times 10^{4} \mathrm{H} 1975$ and $2 \times 10^{6}$ PLTs per well for the Exp_HP group, and the upper chambers were added with $1 \times 10^{4}$ HUVECs plus $0.5 \mathrm{ml}$ RPMI-1640 medium per well for all three groups. The upper chambers were taken out following incubation for $24 \mathrm{~h}$ at $37^{\circ} \mathrm{C}$, the culture medium was drained and cells were washed with PBS. Cells were subsequently fixed with 4\% PFA for $30 \mathrm{~min}$ at room temperature, and then stained in the dark with $10 \%$ crystal violet for $30 \mathrm{~min}$ at room temperature. Stained cells were counted in five randomly selected fields using a fluorescence microscope (DMI6000B; Leica Microsystems, Inc.) in the bright field (magnification, $\mathrm{x} 200$ ), and the number of migrated cells were counted using ImageJ software.

Tube formation assay. Cell suspension containing $5 \times 10^{5}$ HUVECs $(2 \mathrm{ml})$ was added to a 6-well plate, and the cell supernatant was replaced with RPMI-1640 medium, SN_H, and SN_HP following incubation for $24 \mathrm{~h}$ at $37^{\circ} \mathrm{C}$ to form the control, Exp_H and Exp_HP groups. Following incubation for an additional $24 \mathrm{~h}$ at $37^{\circ} \mathrm{C}$, HUVECs were harvested and diluted to $1 \times 10^{5}$ cells $/ \mathrm{ml}$ using RPMI-1640 medium supplemented with 10\% FBS. A total of $60 \mu \mathrm{l}$ Matrigel $(8-12 \mathrm{mg} / \mathrm{ml}$; cat. no. 356234; Corning, Inc.) per well was added into a 96 -well plate at $4^{\circ} \mathrm{C}$ and solidified at $37^{\circ} \mathrm{C}$ for 30-45 min. Subsequently, HUVEC suspensions were plated at a concentration of $1 \times 10^{4}$ cells/well in a 96-well plate coated with Matrigel. Tube formation of HUVECs was observed under an inverted light microscope (magnification, $\mathrm{x} 40$; CKX41; Olympus Corporation) following incubation for 3 and $9 \mathrm{~h}$ at $37^{\circ} \mathrm{C}$.

ELISA detection. Grouping design and cell culture conditions were the same as those used for cell cycle analysis. Briefly, the culture medium was replaced with RPMI-1640 medium without FBS on the 3rd day. Following incubation for $16 \mathrm{~h}$ at $37^{\circ} \mathrm{C}$, VEGF expression in the supernatant of the upper 

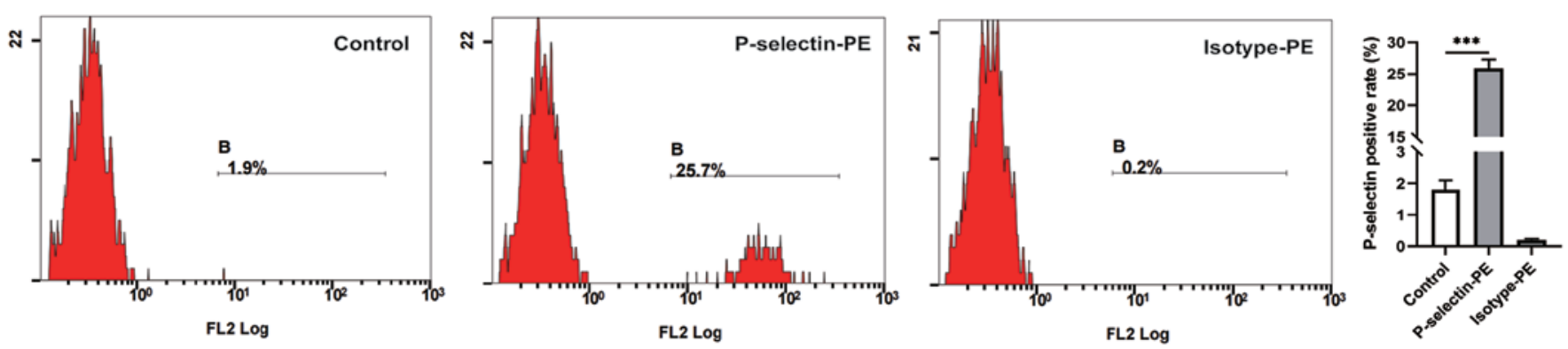

Figure 1. Measurement of P-selectin positivity on platelets activated by H1975 supernatant via flow cytometric analysis. Although three groups are included in the figure, one of them is an isotype control, which was used to eliminate background staining caused by non-specific binding of the antibody to the cell surface. Therefore, the isotype control group was not included in the analysis. ${ }^{* * *} \mathrm{P}<0.001$. PE, phycoerythrin.

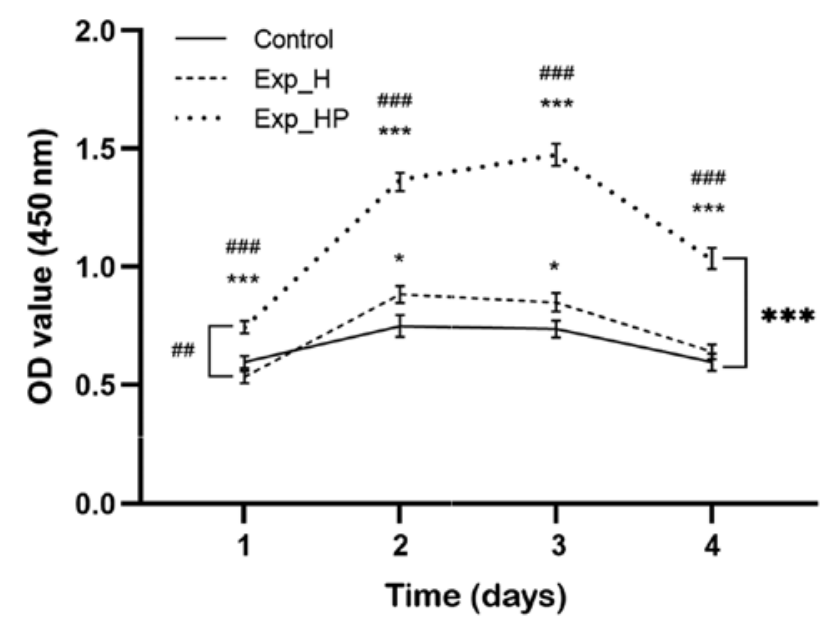

Figure 2. HUVEC activity measured via the Cell Counting Kit- 8 assay at $450 \mathrm{~nm}$. Control: Single cultured HUVECs, Exp_H: HUVECs co-cultured with H1975 cells supernatant and Exp_HP: HUVECs co-cultured with supernatant derived from $\mathrm{H} 1975$ cells co-cultured with platelets. ${ }^{*} \mathrm{P}<0.05$, ${ }^{* * * *} \mathrm{P}<0.001$ vs. control; ${ }^{\# \#} \mathrm{P}<0.01,{ }^{\# \#} \mathrm{P}<0.001$ vs. Exp_H. HUVEC, human umbilical vein endothelial cell; OD, optical density.

chamber was quantitatively measured using the human VEGF ELISA kit (cat. no. H044-1; Nanjing Jiancheng Bioengineering Institute).

Western blot analysis. Grouping design and cell culture conditions were the same as those used for cell cycle analysis. Briefly, the culture medium was replaced with RPMI-1640 medium without FBS on the third day. Following incubation for $16 \mathrm{~h}$ at $37^{\circ} \mathrm{C}$, VEGFR2 expression in the lysate of HUVECs was detected via western blotting. HUVECs were lysed in RIPA lysis buffer (cat. no. P00138, Beyotime Institute of Biotechnology). After quantification by Bradford assay, the protein samples were mixed with $5 \mathrm{X}$ loading buffer. Equal amounts of protein (20-30 $\mu \mathrm{g} /$ lane) were separated using $10 \%$ SDS-PAGE and transferred onto nitrocellulose membranes (Pall Corporation). The membranes were blocked using $5 \%$ skimmed milk for $1 \mathrm{~h}$ at room temperature and incubated with primary antibodies against VEGFR2 (cat. no. ab134191; 1:3,000 dilution; Abcam) and $\beta$-actin (cat. no. TA-09; 1:1,000 dilution; OriGene Technologies, Inc.) overnight at $4^{\circ} \mathrm{C}$. After incubated with the appropriate horseradish peroxidase-labeled IgG (cat. no. A0208, targeting anti-VEGFR2; 1:10,000 dilution; Beyotime Institute of Biotechnology; cat. no.ZB-2205, targeting anti- $\beta$-actin; 1:10,000 dilution; OriGene Technologies, Inc.) at room temperature for $1 \mathrm{~h}$, the proteins were detected with enhanced chemiluminescence reagent (lot, 21064112; Biosharp Life Sciences). TBS with Tween-20 (0.05\%) was used for membrane washing. The Electrophoresis Gel Imaging Analysis System and integrated analysis software (version 1.00.0018, GV 6000plus; Guangzhou Biolight Biotechnology, Ltd.) was used to detect the immunoreactive protein.

Statistical analysis. Statistical analysis was performed using SPSS 23.0 software (IBM Corp.). All experiments were performed in triplicate and data are presented as the mean \pm SD. Unpaired Student's t-test was used to compare the expression rate of P-selectin between the group of P-selectin-PE and the control group, while one-way ANOVA followed by Newman-Keuls post hoc test were used to compare differences between three groups. $\mathrm{P}<0.05$ was considered to indicate a statistically significant difference.

\section{Results}

PLTs were activated by $H 1975$ cells. PLT activation is a key step in the crosstalk between tumor cells and PLTs (24). In the present study, P-selectin expression was detected on the PLT surface via flow cytometry. Fig. 1 depicts the different levels of the PLT activation marker, P-selectin, among the three groups. P-selectin expression was higher in PLTs incubated with SN_H (P-selectin-PE: $25.9 \pm 1.4 \%$ ) compared with the control group $(1.8 \pm 0.3 \%, \mathrm{P}<0.001)$, suggesting that PLTs can be activated by H1975 cells.

HUVEC activity improves following co-culture with PLTs and H1975 cells. The CCK-8 assay was performed to determine whether co-culture with PLTs enhances H1975 cell improvement in HUVEC activity. The activity of HUVECs in the Exp_H group exhibited a slight increase compared with the control group, while HUVECs in the Exp_HP group exhibited a significant increase compared with the Exp_H $(\mathrm{P}<0.01)$ and control group $(\mathrm{P}<0.001)$, respectively (Fig. 2). Further analysis results showed that the activity of HUVECs in the Exp_HP group was higher than that of the Exp_H at all four timepoints $(\mathrm{P}<0.001)$. Similarly, the activity of Exp_HP group was stronger than that of the control at all timepoints $(\mathrm{P}<0.001)$; however, the activity of the Exp_H group was higher than that of the control only at day 2 and day $3(\mathrm{P}<0.05)$. Notably, 
Control

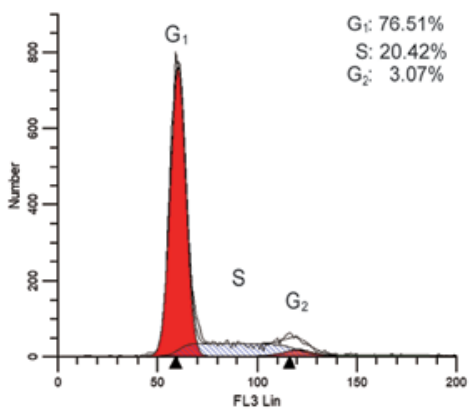

Exp_H

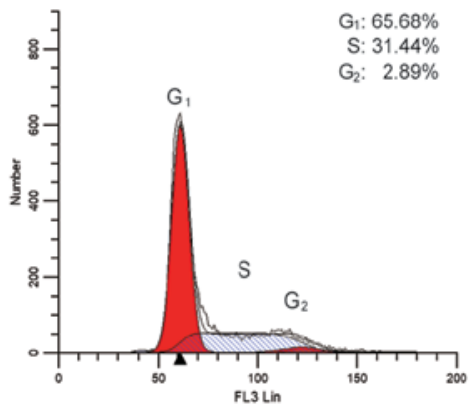

Exp_HP

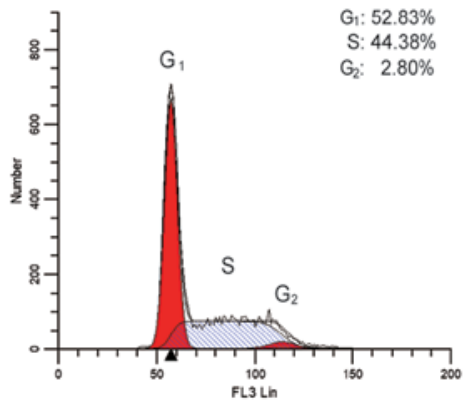

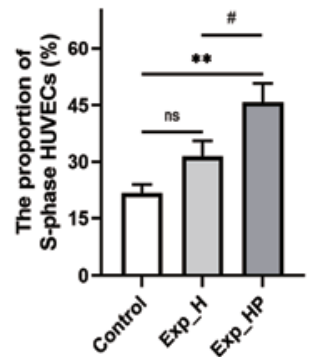

Figure 3. Flow cytometric analysis of HUVEC cycles among the three groups. Control: Single cultured HUVECs, Exp_H: HUVECs co-cultured with H1975 cells supernatant and Exp_HP: HUVECs co-cultured with supernatant derived from H1975 cells co-cultured with platelets. ${ }^{* *} \mathrm{P}<0.01 ;{ }^{*} \mathrm{P}<0.05$; HUVEC, human umbilical vein endothelial cell; ns, not significant.

HUVEC activity in the Exp_HP group reached a peak after 3 days of incubation and subsequently decreased. These results suggested that the activity of HUVECs could be improved by PLT-H1975 crosstalk.

Co-culture with PLTs and H1975 cells increases the proportion of S-phase HUVECs. S-phase is an important phase of cell proliferation that reflects the active state of cell proliferation (25). Thus, it was hypothesized that the proportion of S-phase HUVECs would increase following incubation with SN_H or SN_HP. The results demonstrated that the proportion of

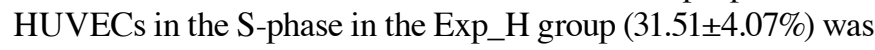
higher compared with the control group $(21.75 \pm 2.34 \%, \mathrm{P}=0.048)$, and the proportion of HUVECs in the S-phase was highest in the Exp_HP group $(45.73 \pm 4.95 \%$; $\mathrm{P}<0.01$ vs. control; $\mathrm{P}<0.05$ vs. Exp_H; Fig. 3). These results suggested that the proliferation of HUVECs could be increased by PLT-H1975 crosstalk.

HUVEC morphology changes following co-culture with PLTs and H1975 cells. It is well-known that tumors have dense blood vessels but incomplete structures (26). Thus, it was hypothesized that the internal structure of HUVECs that proliferate rapidly under SN_HP stimulation may be abnormal. The morphological changes were observed following incubation for 3 days as the results of CCK- 8 assay indicated that HUVECs were at the vigorous growth stage after 3 days of incubation. Fig. 4 depicts the morphological changes of HUVECs observed via transmission electron microscopy. The results demonstrated that more vacuolation of mitochondria was observed in the Exp_H group compared with the control group. In addition, more types of abnormal changes were observed in the Exp_HP group, such as mitochondrial vacuolation, fluffy structure, enlarged nucleus and nuclear membrane deformity. These results suggested that the ultrastructure of HUVECs could be damaged by PLT-H1975 crosstalk.

HUVEC resistance decreases following co-culture with PLTs and H1975 cells. Abnormal structure may decrease the tightness of intercellular connections, and transepithelial electrical resistance can reflect the connections between ECs (27). It was hypothesized that the transepithelial electrical resistance of HUVECs would decrease in the Exp_HP group. Fig. 5 depicts HUVEC resistance on 4 different incubation days. The results demonstrated that resistance continuously increased in the control group, with significant differences between the time points. While the trends of resistance in the Exp_H and Exp_HP groups were different compared with the control group $(\mathrm{P}<0.01)$. Notably, resistance in the Exp_HP and Exp_H groups reached a peak after 2 days of incubation and subsequently decreased at a rapid rate. The difference between the Exp_H and Exp_HP groups was also significant $(\mathrm{P}<0.05)$. These results suggested that the tightness of HUVECs junction could be decreased by PLT-H1975 crosstalk.

SN_HP increases the wound healing capacity of HUVECs. The wound healing and Transwell assays were performed to assess cell migration. The results demonstrated that the relative migration rate of HUVECs was significantly higher in the Exp_HP group, followed by the Exp_H group and the control group (Fig. 6) with significant differences among three groups: $\mathrm{P}<0.05$ for Exp_HP vs. Exp_H and Exp_H vs. control, P<0.01 for Exp_HP vs. control. These results suggested that the wound healing capacity of HUVECs could be promoted by PLT-H1975 crosstalk.

Co-culture with PLTs and H1975 cells improves the migration of HUVECs. The microscopic results of the Transwell migration assay are presented in Fig. 7. Following co-culture with tumor cells and PLTs for $24 \mathrm{~h}$, the migratory ability of HUVECs was significantly higher in the Exp_HP group compared with the control $(\mathrm{P}<0.01)$ and Exp_H groups $(\mathrm{P}<0.05)$, and the migratory ability of HUVECs was significantly higher in the Exp_H group compared with the control group $(\mathrm{P}<0.05)$. These results were consistent with those of the wound healing assay. These results suggested that the migration capacity of HUVECs could be improved by PLT-H1975 crosstalk.

SN_HP promotes the tube-forming ability of HUVECs. Based on the aforementioned results, it was revealed that H1975-PLT crosstalk can promote the proliferation and migration of HUVECs. Thus, it was hypothesized that crosstalk may improve the tube formation ability of HUVECs. Fig. 8 demonstrates the tube formation ability indicated by the junction number, mesh number and total length of HUVECs among the three groups. The tube-forming ability increased in each group with time. Furthermore, the function of HUVECs in the Exp_HP group was significantly better compared with the control group 

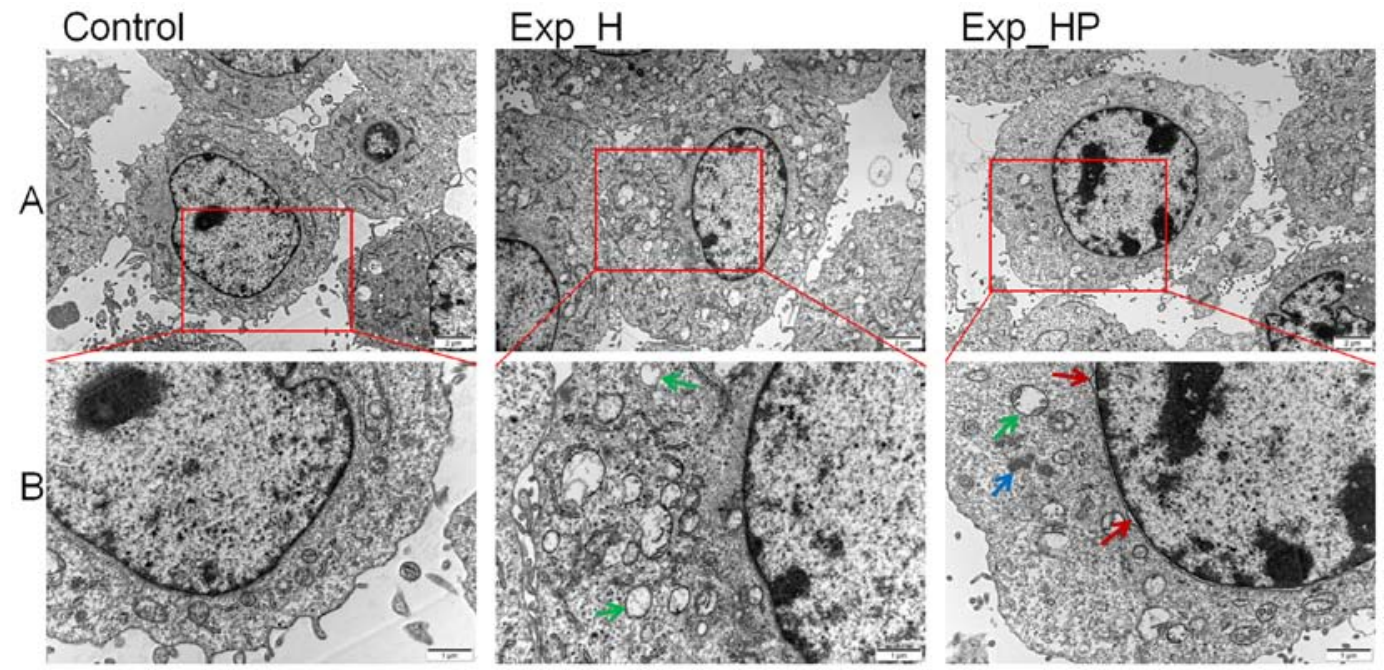

Figure 4. Transmission electron microscopy morphological changes of HUVECs among the three groups. (A) Magnification, x8,000. (B) Magnification, x20,000 . Control: Single cultured HUVECs, Exp_H: HUVECs co-cultured with H1975 cells supernatant and Exp_HP: HUVECs co-cultured with supernatant derived from H1975 cells co-cultured with platelets. Red box: Area to be magnified. Green arrow depicts vacuolation of mitochondria, blue arrow depicts rough endoplasmic reticulum dilatation and red arrow depicts nuclear membrane rupture. HUVEC, human umbilical vein endothelial cell.

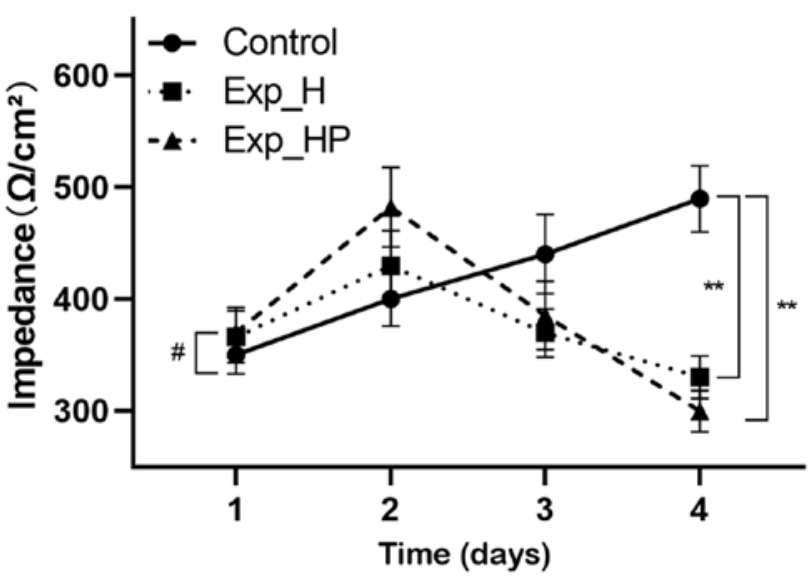

Figure 5. Transepithelial electrical resistance of HUVECs at 4 different incubation days among the three groups. Control: Single cultured HUVECs, Exp_H: HUVECs co-cultured with H1975 cells supernatant and Exp_HP HUVECs co-cultured with supernatant derived from H1975 cells co-cultured with platelets. ${ }^{* *} \mathrm{P}<0.01 ;{ }^{\prime} \mathrm{P}<0.05$. HUVEC, human umbilical vein endothelial cell.

following 3 and $9 \mathrm{~h}$ of incubation ( $\mathrm{P}<0.05)$, especially for junction number $3 \mathrm{~h}$ after incubation $(\mathrm{P}<0.01)$ and mesh number $9 \mathrm{~h}$ after incubation $(\mathrm{P}<0.001)$. Similarly, the tube-forming ability in the Exp_HP group was significantly higher compared with the Exp_H group following 3 and $9 \mathrm{~h}$ of incubation $(\mathrm{P}<0.05)$, especially for mesh number $9 \mathrm{~h}$ after incubation $(\mathrm{P}<0.01)$. However, no significant differences were observed between the Exp_H and control groups except for mesh number $9 \mathrm{~h}$ after incubation $(\mathrm{P}<0.05)$. These results suggested that the tube-forming ability of HUVECs could be increased by PLT-H1975 crosstalk.

Co-culture with PLTs and H1975 cells increases VEGF and VEGFR2 expression levels in HUVECs. VEGF is highly specific and the combination of VEGF and VEGFR2 is the key mechanism that induces tumor angiogenesis (28). It was hypothesized that H1975-PLT crosstalk may enhance VEGF and VEGFR2 expression levels in HUVECs. Fig. 9 depicts VEGF content measured by ELISA and VEGFR 2 expression detected via western blotting. High VEGF expression was observed in the Exp_HP group $(29.32 \pm 3.25 \mathrm{ng} / \mathrm{l})$, which was significantly higher compared with the Exp_H $(19.93 \pm 1.71 \mathrm{ng} / \mathrm{l}$; $\mathrm{P}<0.01)$ and control $(15.86 \pm 0.73 \mathrm{ng} / \mathrm{l} ; \mathrm{P}<0.001)$ groups. In addition, VEGF expression was significantly higher in the Exp_H group compared with the control group $(\mathrm{P}<0.05)$. Similarly, the highest expression of VEGFR2 was observed in the Exp_HP group, followed by the Exp_H group and the control group, with significant differences among three groups: $\mathrm{P}<0.05$ for Exp_HP vs. Exp_H, P $<0.01$ for Exp_H vs. control, and $\mathrm{P}<0.001$ for Exp_HP vs. control. These results suggested that the expression levels of VEGF and VEGFR2 in HUVECs could be improved by PLT-H1975 crosstalk.

\section{Discussion}

Angiogenesis is the central marker of tumors and is key for the progression and metastasis of solid tumors (6). HUVECs are a classic model system to study angiogenesis (29). Previous studies have demonstrated that tumor cells can improve the proliferation and migration of VECs, and promote angiogenesis to a certain extent (30-32); activated PLTs are also involved in this process $(16,17,33)$. However, the effect of co-culture of tumor cells and PLTs on the proliferation, migration and angiogenesis of HUVECs remains unknown. To the best of our knowledge, the present study was the first to investigate H1975-PLT crosstalk on the properties of HUVECs based on CCS. The results demonstrated that the activity of HUVECs significantly enhanced following co-culture with tumor cells and PLTs, which can help illustrate the mechanism of tubular formation of VECs around cancer cells.

Angiogenesis is induced when the tumor grows to $2 \mathrm{~mm}$ in diameter; otherwise tumor cells die due to a lack of nutrition (34). VECs serve a key role in tumor angiogenesis (32). To determine the effect of tumor-PLT crosstalk on the ability of VECs, the present study assessed the proliferation, migration 


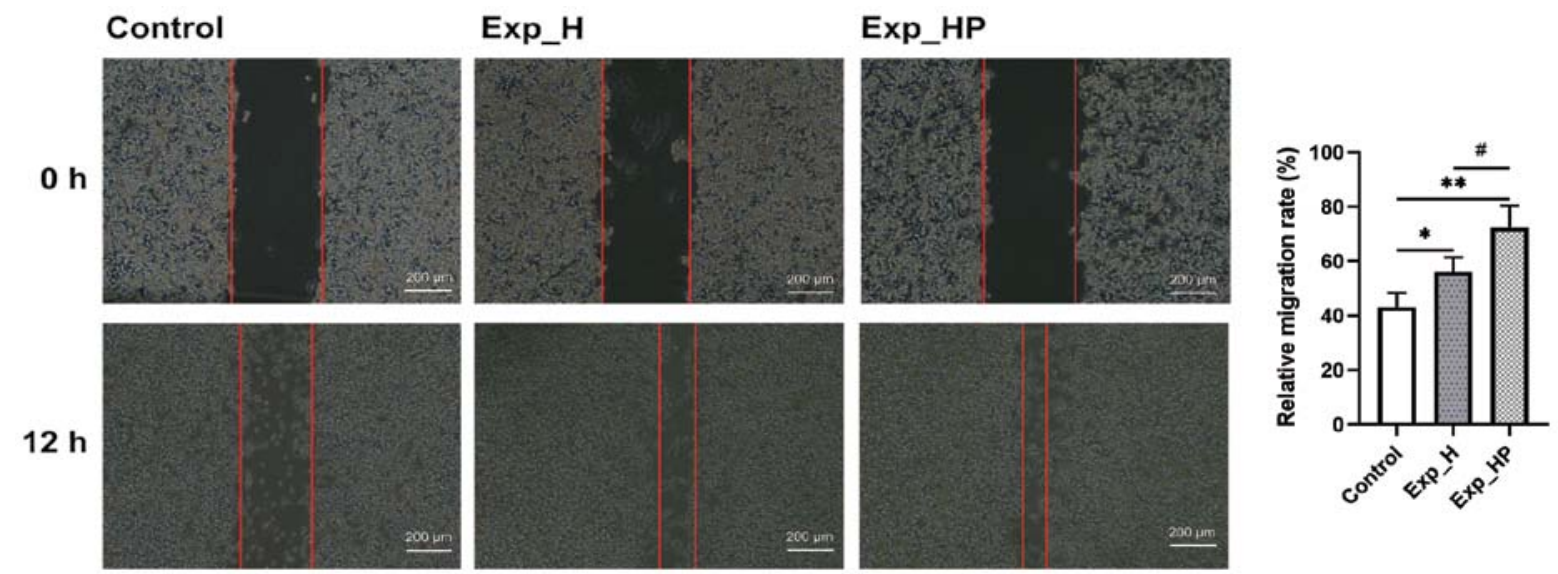

Figure 6. Effect of co-cultured supernatant on the wound healing ability of HUVECs among the three groups. Left: Representative photomicrographs of the wound healing assay at 0 and $12 \mathrm{~h}$. Right: Bar chart of the relative migration rate of HUVECs. Control: Single cultured HUVECs, Exp_H: HUVECs co-cultured with H1975 cells supernatant and Exp_HP: HUVECs co-cultured with supernatant derived from H1975 cells co-cultured with platelets. " $\mathrm{P}<0.05$; ${ }^{* *} \mathrm{P}<0.01 ;{ }^{\#} \mathrm{P}<0.05$. HUVEC, human umbilical vein endothelial cell.
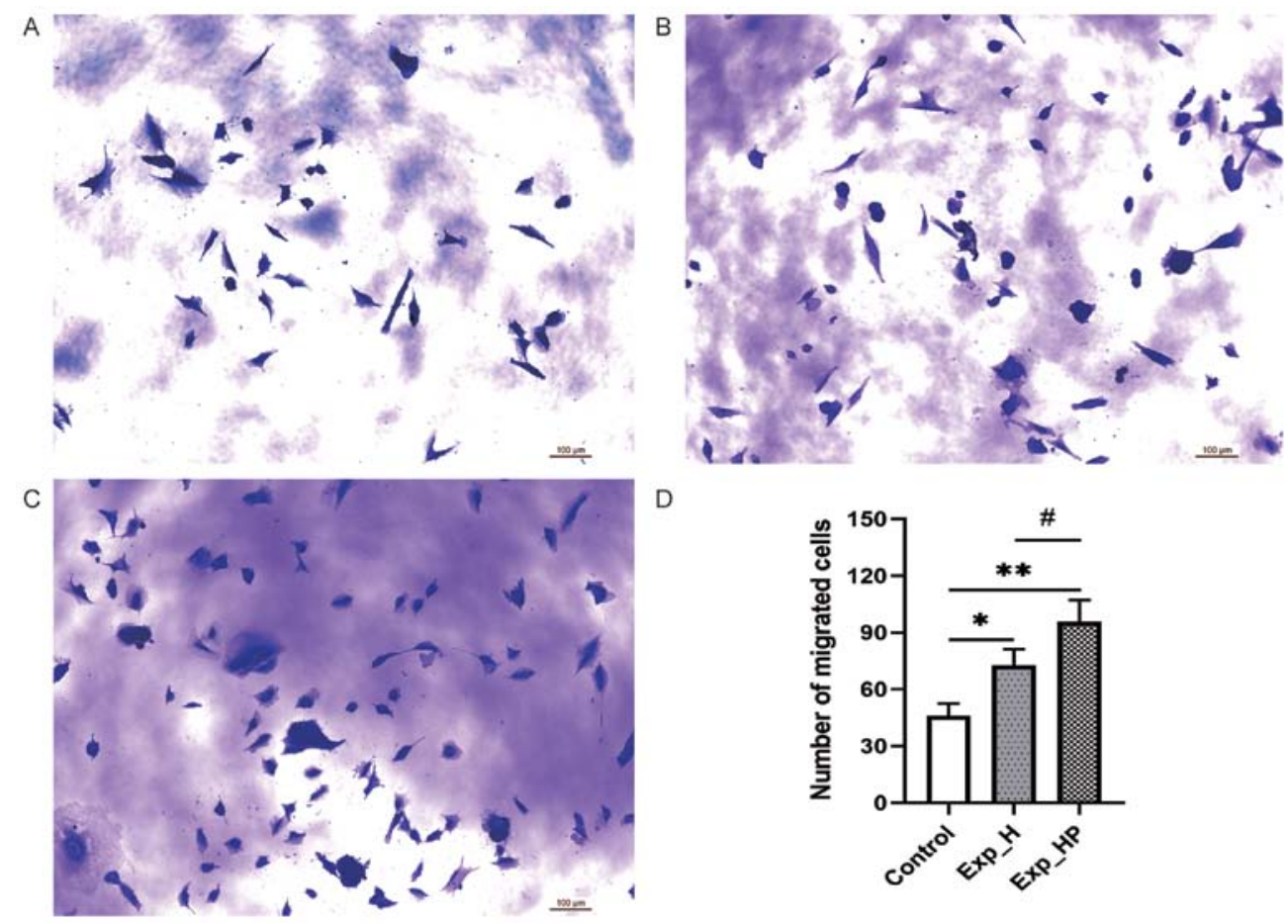

Figure 7. Effect of co-cultured supernatant on the migratory ability of HUVECs among the three groups. (A) Control: Single cultured HUVECs, (B) Exp_H: HUVECs co-cultured with H1975 cells supernatant and (C) Exp_HP: HUVECs co-cultured with supernatant derived from H1975 cells co-cultured with platelets. (D) Histogram of numbers of migrated HUVECs among three groups. ${ }^{*} \mathrm{P}<0.05 ;{ }^{* *} \mathrm{P}<0.01 ;{ }^{*} \mathrm{P}<0.05$. HUVEC, human umbilical vein endothelial cell.

and tube formation abilities of HUVECs and their molecular basis.

Proliferation reflects the basic functional state of HUVECs. The present study investigated the effect of tumor-PLT crosstalk on the proliferation of HUVECs by analyzing cell activity, the proportion of S-phase cells, ultrastructure and cell resistance. The results demonstrated that H1975-PLT crosstalk significantly promoted HUVEC proliferation, with a higher OD value detected via CCK-8 and a higher proportion of S-phase cells in the cell cycle. However, more HUVEC ultrastructure anomalies and a lower degree of tight connections were observed between HUVECs following co-culture with H1975 cells and PLTs. These results are consistent with the features of blood vessels in the tumor microenvironment, which are dense, disordered and incomplete (35-38). It is assumed that if the proliferation of VECs is blocked or weakened, this reduces tumor angiogenesis, which helps control tumor growth and metastasis (9). Activated PLTs can directly stimulate the proliferation of tumor cells and enhance the proliferation of VECs $(13,39)$. Thus, inhibition of PLT activation, particularly tumor cell-induced PLT activation, may be a potential target to prevent tumor-PLT crosstalk and inhibit the proliferation of HUVECs.

Migration of VECs serves an important role in angiogenesis (40). The present study performed wound healing and Transwell assays to determine the effect of H1975-PLT crosstalk on the migratory ability of HUVECs. The results demonstrated 

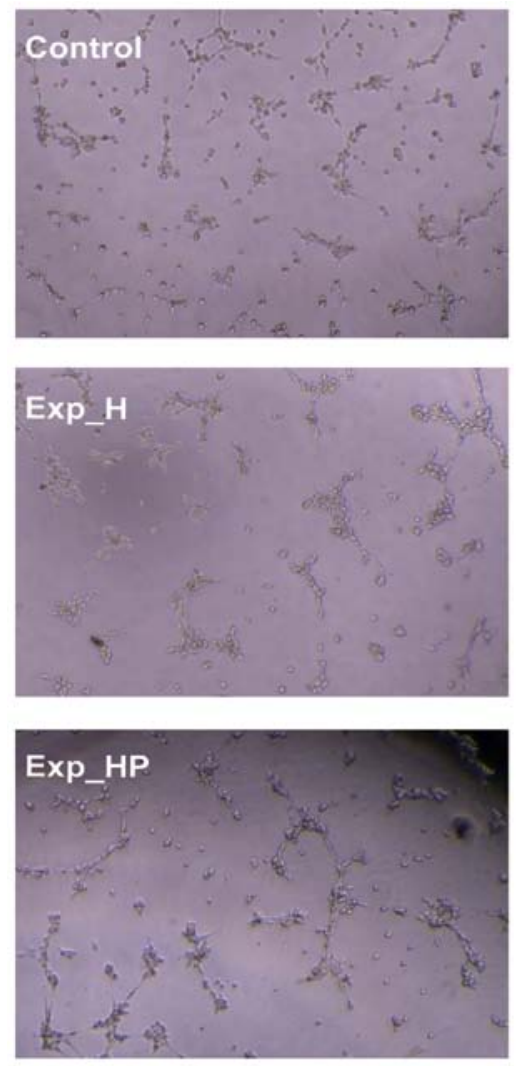

$3 h$
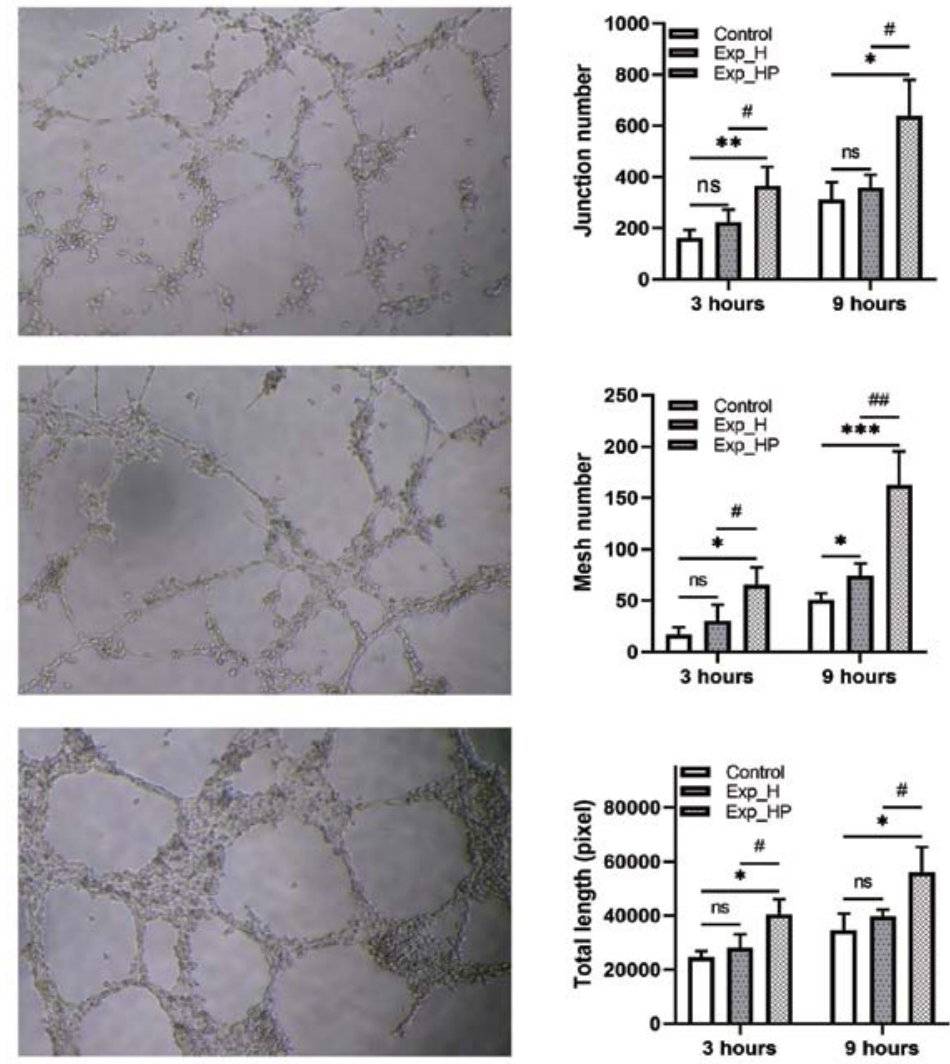

Figure 8. Effect of co-cultured supernatant on tube formation of HUVECs among the three groups. Left: Representative photomicrographs of the tube formation assays at 3 and $9 \mathrm{~h}$. Right: Bar charts of the junction number, mesh number and total length of HUVECs. Control: Single cultured HUVECs, Exp_H: HUVECs co-cultured with H1975 cells supernatant and Exp_HP: HUVECs co-cultured with supernatant derived from H1975 cells co-cultured with platelets ${ }^{*} \mathrm{P}<0.05 ;{ }^{* *} \mathrm{P}<0.01 ;{ }^{* * *} \mathrm{P}<0.001 ;{ }^{\#} \mathrm{P}<0.05 ;{ }^{\# \#} \mathrm{P}<0.01$. HUVEC, human umbilical vein endothelial cell; ns, no significance.
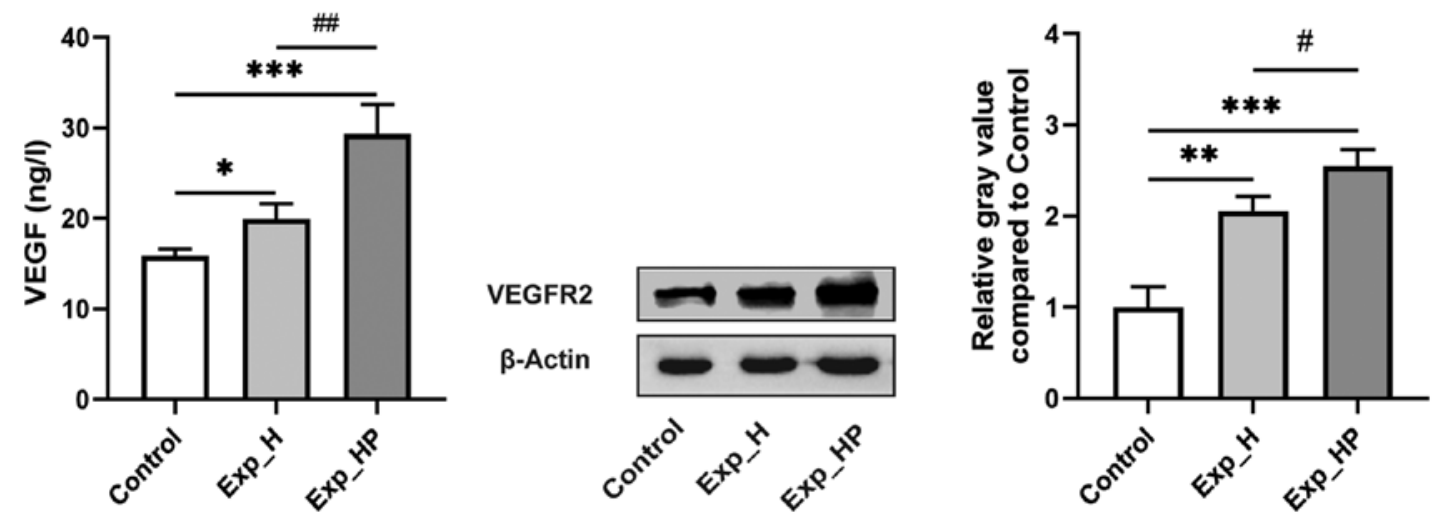

Figure 9. VEGF content in HUVECs among the three groups. Left: VEGF content was measured via ELISA. Middle: VEGFR2 expression was detected via western blotting. Right: Relative gray value of VEGFR2 expression compared with control. Control: Single cultured HUVECs, Exp_H: HUVECs co-cultured with H1975 cells supernatant and Exp_HP: HUVECs co-cultured with supernatant derived from $\mathrm{H} 1975$ cells co-cultured with platelets. "P $<0.05$; ${ }^{* *} \mathrm{P}<0.01$; ${ }^{* * *} \mathrm{P}<0.001 ;{ }^{*} \mathrm{P}<0.05 ;{ }^{\# \#} \mathrm{P}<0.01$. VEGF, vascular endothelial growth factor; HUVEC, human umbilical vein endothelial cell; VEGFR2, VEGF receptor 2.

that the migratory ability of HUVECs was significantly stronger in the Exp_HP group compared with the control group, suggesting that more HUVEC stimulation may be produced in the Exp_HP group compared with the Exp_H and control groups. The results of the present study were consistent with previous findings $(12,30)$. The results of the Transwell assay demonstrated that inhibition of PLT activation may be a potential target to inhibit the migration of HUVECs induced by H1975-PLT crosstalk.
According to the aforementioned research results, tumor-PLT crosstalk could promote HUVECs proliferation and migration. It was speculated that tumor-PLT crosstalk can enhance the tube formation ability of HUVECs. Tube-forming experiments are a rapid and quantifiable method to measure angiogenesis in vitro (41). The present study investigated the effect of H1975-PLT crosstalk on HUVEC angiogenesis using junction number, mesh number and total length as indicators of 
tube-forming ability, and the results of the present study confirmed that the tube-forming ability of HUVECs could be promoted by H1975-PLT crosstalk. These results are consistent with previous findings, suggesting that PLTs can promote tube formation by adhering to ECs or releasing PLT microparticles $(16,42)$. Thus, PLTs activated by tumors may upregulate the expression levels of P-selectin and GIIbIIIa and release more PLT microparticles to promote tube formation $(20,43)$. Taken together, these results suggest that controlling H1975-PLT crosstalk can inhibit angiogenesis and be used as a therapeutic target for tumor metastasis.

Several factors affect the proliferation, migration and tube-forming abilities of HUVECs with complex mechanisms, and the combination of VEGF and VEGFR2 is a key mechanism that induces tumor angiogenesis (28). The present study measured VEGF content in the HUVEC supernatant and detected VEGFR2 expression in the lysate of HUVECs. The results demonstrated that both VEGF and VEGFR2 expression levels were highest in the Exp_HP group, which suggests that H1975-PLT crosstalk may not only stimulate the expression levels of VEGF and VEGFR2 in HUVECs (33) but also promote their combination. Thus, H1975-PLT crosstalk may enhance the proliferation, migration and tube formation of HUVECs. Notably, the increased levels of VEGF may mediate the tumor promotion of PLTs. This result is similar to a previous study that reported that PLTs from patients with glioblastoma can promote angiogenesis of tumor ECs and exhibit increased VEGF content and release (17).

The interaction between H1975 cells and PLTs plays an important role in tumor angiogenesis by affecting the characteristics of ECs, which suggests that inhibition of H1975-PLT crosstalk may be a potential target for the treatment of non-smoking patients with lung adenocarcinoma. However, further studies are required to effectively interfere with tumor-PLT crosstalk without affecting normal coagulation function. Thus, prospective studies will investigate the effect of crosstalk between A549 cells and PLTs on the properties of HUVECs, such as proliferation, migration and tube formation, and evaluate antitumor Chinese medicine, which can interfere with the interaction between tumor cells and PLTs $(44,45)$.

In conclusion, the results of the present study demonstrated that the proliferation, migration and tube-forming abilities of HUVECs co-cultured with H1975 and PLTs were significantly higher compared with HUVECs cultured alone and better than HUVECs co-cultured with only H1975. Taken together, these results suggest that tumor cells interacting with PLTs may play an important role in tumor angiogenesis by affecting or mediating changes in the properties of ECs.

\section{Acknowledgements}

The authors would like to thank Mr. Ruishan Liu (Anhui Medical University, Hefei, China), Dr Ying Cao (Anhui University of Chinese Medicine, Hefei, China), Dr Hui Cheng (Anhui University of Chinese Medicine, Hefei, China) for their technical assistance.

\section{Funding}

The present study was funded by the Opening Foundation of Key Laboratory of Xin'an Medicine, Ministry of Education of
Anhui University of Chinese Medicine (grant no. 2020xayx05) and the Scientific Research Foundation of Education Department of Anhui Province (grant nos. KJ2019A046 and SK2020A0236).

\section{Availability of data and materials}

The datasets used and analyzed during the current study are available from the corresponding author upon reasonable request.

\section{Authors' contributions}

BL conceived the study and XD, JZ, TZ, DP and QL designed the present study. BL, XD, XT, TZ and JZ performed the experiments and statistical analysis. $\mathrm{BL}$ and $\mathrm{XD}$ drafted the initial manuscript. BL, XD, XT, JZ, TZ and QL were involved in reviewing and editing the manuscript for important intellectual content. BL, DP and QL supervised the study and provided funding. BL and QL confirm the authenticity of all the raw data. All authors have read and approved the final manuscript.

\section{Ethics approval and consent to participate}

The present study was approved by the Scientific Research Ethics Committee of the First Affiliated Hospital of Anhui University of Traditional Chinese Medicine (approval no. 2020AHZY-01; Hefei, China). All participants provided oral consent, since the expected risk was low for qualified participants contributing $10 \mathrm{ml}$ venous blood in the hospital, this method of obtaining consent was approved by the Scientific Research Ethics Committee and volunteers chose oral rather than written consent.

\section{Patient consent for publication}

Not applicable.

\section{Competing interests}

The authors declare that they have no competing interests.

\section{References}

1. Bray F, Ferlay J, Soerjomataram I, Siegel RL, Torre LA and Jemal A: Global cancer statistics 2018: GLOBOCAN estimates of incidence and mortality worldwide for 36 cancers in 185 countries. CA Cancer J Clin 68: 394-424, 2018.

2. Yu C, Chen F, Wang X, Cai Z, Yang M, Zhong Q, Feng J, Li J, Shen $C$ and Wen Z: Pin2 telomeric repeat factor 1-interacting telomerase inhibitor 1 (PinX1) inhibits nasopharyngeal cancer cell stemness: Implication for cancer progression and therapeutic targeting. J Exp Clin Cancer Res 39: 31, 2020.

3. Okudela K, Matsumura M, Arai H and Woo T: The nonsmokers' and smokers' pathways in lung adenocarcinoma: Histological progression and molecular bases. Cancer Sci: Jun 18, 2021 (Epub ahead of print). doi: 10.1111/cas.15031

4. Seow WJ, Shu XO, Nicholson JK, Holmes E, Walker DI, Hu W, Cai Q, Gao YT, Xiang YB, Moore SC, et al: Association of untargeted urinary metabolomics and lung cancer risk among never-smoking women in China. JAMA Netw Open 2: e1911970, 2019.

5. Carmeliet P and Jain RK: Molecular mechanisms and clinical applications of angiogenesis. Nature 473: 298-307, 2011.

6. Quail DF and Joyce JA: Microenvironmental regulation of tumor progression and metastasis. Nat Med 19: 1423-1437, 2013. 
7. Aggarwal C, Somaiah $\mathrm{N}$ and Simon G: Antiangiogenic agents in the management of non-small cell lung cancer: Where do we stand now and where are we headed? Cancer Biol Ther 13: 247-263, 2012.

8. Rivera LB and Bergers G: CANCER. Tumor angiogenesis, from foe to friend. Science 349: 694-695, 2015.

9. Al-Abboodi M, An R, Weber M, Schmid R, Klausing A, Horch RE, Boos AM and Kengelbach-Weigand A: Tumor type dependent effects on the angiogenic abilities of endothelial cells in an in vitro rat cell model. Oncol Rep 42: 350-360, 2019.

10. Perut F, Roncuzzi L,Zini N, Massa A and Baldini N: Extracellular nanovesicles secreted by human osteosarcoma cells promote angiogenesis. Cancers (Basel) 11: 779, 2019.

11. Ghorbanian M, Babashah S and Ataei F: The effects of ovarian cancer cell-derived exosomes on vascular endothelial growth factor expression in endothelial cells. EXCLI J 18: 899-907, 2019.

12. Li N: Platelets in cancer metastasis: To help the 'villain' to do evil. Int J Cancer 138: 2078-2087, 2016.

13. Chiodoni C, Iezzi M, Guiducci C, Sangaletti S, Alessandrini I, Ratti C, Tiboni F, Musiani P, Granger DN and Colombo MP. Triggering CD40 on endothelial cells contributes to tumor growth. J Exp Med 203: 2441-2450, 2006.

14. Gay LJ and Felding-Habermann B: Contribution of platelets to tumour metastasis. Nat Rev Cancer 11: 123-134, 2011.

15. Borsig L: The role of platelet activation in tumor metastasis Expert Rev Anticancer Ther 8: 1247-1255, 2008.

16. Kim HK, Song KS, Chung JH, Lee KR and Lee SN: Platelet microparticles induce angiogenesis in vitro. Br J Haematol 124 376-384, 2004

17. Di Vito C, Navone SE, Marfia G, Abdel Hadi L, Mancuso ME, Pecci A, Crisà FM, Berno V, Rampini $\mathrm{P}$, Campanella R, et al: Platelets from glioblastoma patients promote angiogenesis of tumor endothelial cells and exhibit increased VEGF content and release. Platelets 28: 585-594, 2017.

18. Li R, Ren M, Chen N, Luo M, Deng X, Xia J, Yu G, Liu J, He B Zhang X, et al: Presence of intratumoral platelets is associated with tumor vessel structure and metastasis. BMC Cancer 14: 167, 2014.

19. Campanella R, Guarnaccia L, Cordiglieri C, Trombetta E, Caroli M, Carrabba G, La Verde N, Rampini P, Gaudino C, Costa A, et al: Tumor-educated platelets and angiogenesis in glioblastoma: Another brick in the wall for novel prognostic and targetable biomarkers, changing the vision from a localized tumor to a systemic pathology. Cells 9: 294, 2020.

20. Haemmerle M, Stone RL, Menter DG, Afshar-Kharghan V, Sood AK: The platelet lifeline to cancer: Challenges and opportunities. Cancer Cell 33: 965-983, 2018.21.

21. Carrim N, Arthur JF, Hamilton JR, Gardiner EE, Andrews RK, Moran N, Berndt MC and Metharom P: Thrombin-induced reactive oxygen species generation in platelets: A novel role for protease-activated receptor 4 and GPIb $\alpha$. Redox Biol 6: 640-647, 2015.

22. Mercatali L, La Manna F, Miserocchi G, Liverani C, De Vita A, Spadazzi C, Bongiovanni A, Recine F, Amadori D, Ghetti M, et al: Tumor-stroma crosstalk in bone tissue: The osteoclastogenic potential of a breast cancer cell line in a co-culture system and the role of EGFR inhibition. Int J Mol Sci 18: E1655, 2017.

23. Kraya R, Komin A and Searson P: On chip bioelectric impedance spectroscopy reveals the effect of p-glycoprotein efflux pumps on the paracellular impedance of tight junctions at the blood-brain barrier. IEEE Trans Nanobioscience 15: 697-703, 2016.

24. LI S: CHEN ZB, LÜ M, ZHU Y: Current status and future of drugs targeting platelets-tumor cells interactions. Yao Xue Xue Bao 2: 360-367, 2021 (In Chinese)

25. Mazurek A, Luo W, Krasnitz A, Hicks J, Powers RS and Stillman B: DDX5 regulates DNA replication and is required for cell proliferation in a subset of breast cancer cells. Cancer Discov 2: 812-825, 2012.

26. Jain RK: Antiangiogenesis strategies revisited: From starving tumors to alleviating hypoxia. Cancer Cell 26: 605-622, 2014.

27. Yang YF, Meng YY, Ye J, Xia XJ, Li L, Dong WJ, Wang HL and Liu YL: Co-culture of human breast adenocarcinoma cells and human umbilical vein endothelial cells to mimic in vivo tumor microenvironment. Yao Xue Xue Bao 3: 403-409, 2018 (In Chinese).
28. Khan KA and Kerbel RS: Improving immunotherapy outcomes with anti-angiogenic treatments and vice versa. Nat Rev Clin Oncol 15: 310-324, 2018

29. Lin X, Qiu W, Xiao Y, Ma J, Xu F, Zhang K, Gao Y, Chen Q, $\mathrm{Li}$ Y, Li H, et al: MiR-199b-5p suppresses tumor angiogenesis mediated by vascular endothelial cells in breast cancer by targeting ALK1. Front Genet 10: 1397, 2020.

30. Ishihara S, Onoda N, Noda S, Asano Y, Tauchi Y, Morisaki T, Kashiwagi S, Takashima T and Ohira M: Sorafenib inhibits vascular endothelial cell proliferation stimulated by anaplastic thyroid cancer cells regardless of BRAF mutation status. Int J Oncol 55: 1069-1076, 2019.

31. Kaneda H, Arao T, Matsumoto K, De Velasco MA, Tamura D, Aomatsu K, Kudo K, Sakai K, Nagai T, Fujita Y, et al: Activin A inhibits vascular endothelial cell growth and suppresses tumour angiogenesis in gastric cancer. Br J Cancer 105: 1210-1217, 2011.

32. Guo D, Xu S, Wang N, Jiang H, Huang Y, Jin X, Xue B, Zhang C and Zhu X: Prodrug-embedded angiogenic vessel-targeting nanoparticle: A positive feedback amplifier in hypoxia-induced chemo-photo therapy. Biomaterials 144: 188-198, 2017.

33. Frezzetti D, Gallo M, Roma C, D'Alessio A, Maiello MR, Bevilacqua S, Normanno N and De Luca A: Vascular endothelial growth factor a regulates the secretion of different angiogenic factors in lung cancer cells. J Cell Physiol 231: 1514-1521, 2016.

34. Hanahan D and Folkman J: Patterns and emerging mechanisms of the angiogenic switch during tumorigenesis. Cell 86: 353-364, 1996.

35. Carmeliet P and Jain RK: Principles and mechanisms of vessel normalization for cancer and other angiogenic diseases. Nat Rev Drug Discov 10: 417-427, 2011.

36. Jain RK: Normalization of tumor vasculature: An emerging concept in antiangiogenic therapy. Science 307: 58-62, 2005.

37. Nagy JA, Chang SH, Shih SC, Dvorak AM and Dvorak HF: Heterogeneity of the tumor vasculature. Semin Thromb Hemost 36: 321-331, 2010

38. Mierke CT: Cancer cells regulate biomechanical properties of human microvascular endothelial cells. J Biol Chem 286: 40025-40037, 2011.

39. Schumacher D, Strilic B, Sivaraj KK, Wettschureck N and Offermanns S: Platelet-derived nucleotides promote tumor-cell transendothelial migration and metastasis via $\mathrm{P} 2 \mathrm{Y} 2$ receptor. Cancer Cell 24: 130-137, 2013.

40. Jiang X, Hu J, Wu Z, Cafarello ST, Di Matteo M, Shen Y, Dong X, Adler H, Mazzone M, Ruiz de Almodovar C, et al: Protein phosphatase 2A mediates YAP activation in endothelial cells upon VEGF stimulation and matrix stiffness. Front Cell Dev Biol 9: 675562,2021

41. Behnammanesh G, Durante ZE, Peyton KJ, Martinez-Lemus LA, Brown SM, Bender SB and Durante W: Canagliflozin inhibits human endothelial cell proliferation and tube formation. Front Pharmacol 10: 362, 2019

42. Pipili-Synetos E, Papadimitriou E and Maragoudakis ME: Evidence that platelets promote tube formation by endothelial cells on matrigel. Br J Pharmacol 125: 1252-1257, 1998.

43. Wassmer SC, Taylor T, Maclennan CA, Kanjala M, Mukaka M, Molyneux ME and Grau GE: Platelet-induced clumping of Plasmodium falciparum-infected erythrocytes from Malawian patients with cerebral malaria-possible modulation in vivo by thrombocytopenia. J Infect Dis 197: 72-78, 2008.

44. Lin WF, Lu JY, Cheng BB and Ling CQ: Progress in research on the effects of traditional Chinese medicine on the tumor microenvironment. J Integr Med 15: 282-287, 2017.

45. Li Q, Chen Y, Zhao D, Yang S, Zhang S, Wei Z, Wang Y, Qian K, Zhao B, Zhu Y, et al: LongShengZhi Capsule reduces carrageenan-induced thrombosis by reducing activation of platelets and endothelial cells. Pharmacol Res 144: 167-180, 2019.

This work is licensed under a Creative Commons

Attribution-NonCommercial-NoDerivatives 4.0 International (CC BY-NC-ND 4.0) License. 